\title{
REFLEXIVIDAD Y CONTEXTO EN EL QUEHACER ANTROPOLÓGICO.
}

\author{
Reflexivity and Context into Anthropological Work \\ Teresa Durán, Noelia Carrasco, Marcelo Berhó ?
}

\section{RESUMEN}

Los cambios sustantivos que se han generado en la disciplina parecen responder a una matriz más o menos similar: los antropólogos toman conciencia de los límites de las teorías que manejan o aplican respecto de determinadas características socioculturales del contexto en el que trabajan. Ante tales desafíos intelectuales tienden a formular propuestas que consideran diferentes a las convencionales en uso, siendo los pares quienes legitiman los aportes que tales propuestas puedan representar, en el campo teórico y/o metodológico.

En este artículo se presenta de un modo sucinto una experiencia de re-enfoque de la antropología formal y particularmente del quehacer antropológico en un contexto dado, un contexto en el cual el antropólogo no es un visitante sino que es estratégicamente, convocado por la sociedad local. La base metodológica de esta experiencia es la reflexividad generada en los últimos diez años a partir de la práctica sistemática de una etnografía reflexiva en el marco de una teoría postestructuralista. Las problemáticas centrales se expresan en interrogantes tales como: ¿̇cómo se reproduce la antropología en contextos distintos a los originarios? la antropología? y y è qué medida el antropólogo re-direcciona los cambios de su quehacer?

\section{Palabras clave}

Reflexividad, contexto, etnografía reflexiva, quehacer antropológico, antropología interactiva

Trabajo recepcionado: Marzo 2005

Trabajo aceptado: Agosto 2005

1. Todos son docentes e investigadores de la Escuela de Antropología y del Centro de Estudios Socioculturales (CES) de la Universidad Católica de Temuco (UCT), Casilla 15 D, Temuco, Chille; tduran@uct.cl; ncar@uct.cl; mberho@uct.cl. 
The recent substantive transformations in the discipline of anthropology seem to respond to a similar impulse: that anthropologists are becoming increasingly conscious of the limits of theories when applied to the socio-cultural specificity of the local contexts in which they work. Faced with such intellectual quandaries, anthropologists tend to formulate alternative proposals whose theoretical and / or methodological legitimacy only their peers are deemed capable of judging.

This article will present an attempt to refocus the tasks of faced by formal anthropology in a particular local context in which the anthropologist is not a visitor but is strategically invited by the local population. The methodological base of this experience is found in the reflections spawned by ten years of the systematic application of a reflexive ethnography, situated within post-structural theory. The central problems addressed are expressed in questions such as: how do you reproduce anthropology in contexts distinct from their original ones, to what degree do such contexts influence anthropology, and to what extent does the anthropologist redirect the development of his / her work?

\section{Key words}

Reflexivity, context, reflexive etnography, anthropological work, interactive anthropolo

\section{INTRODUCCIÓN}

En este artículo, intentaremos formular una aproximación ilustrativa a lo que podríamos denominar el itinerario de la antropología desde su quehacer, como quehacer, en un contexto parlante, tomando como referencia, por un lado, la información formal de que disponemos en relación a como se da cuenta habitualmente este proceso y nuestra propia experiencia de construcción de conocimiento y de advertir su impacto tanto en el contexto como en el proceso inducidor. En principio, podría decirse que un acercamiento de esta naturaleza requeriŕa la información que proveen los propios antropólogos y probablemente sus pares. En nuestra perspectiva, agregaremos a estos dos indicadores, el impacto que las construcciones y quehaceres tienen en la sociedad $y$ particularmente en los sujetos que aprenden antropología. En este sentido, proponemos interpretar el quehacer antropológico como herramienta de socialización de segundo orden, es decir, como definición y redefinición del sujeto, del otro y de los fundamentos mismos del proceso de construcción de conocimiento disciplinario.

\section{METODOLOGÍA}

La mayoría de los antropólogos clásicos recurrieron tácitamente a un sustrato teóricoepistemológico racionalista expresado en la concepción de que la antropología se construye mediante la formulación de "teorías" y se desarrolla a partir de su deconstrucción y reconstrucción. El concepto de teoría se concibe de un modo operacional como "un cuerpo de proposiciones interconexas (hipótesis, generalizaciones) referentes a un área problemática, de cuya factualidad empírica, se proponen dar razón" (Braithwaite, 1953, cit. en Nadel, 1966: 27). Se entenderá que las teorías se orientan a explicar $y$, por tanto, se trataría de 
generalizaciones respecto de las cuales "se siguen lógicamente consecuencias observables" (Nadel, 1966:27).

Según Meyer, el cambio teórico propuesto por Nadel en la teoría estructuralista británica, provocó un impacto importante en la disciplina. Al decir de Meyer, Nadel estaba preocupado del problema de ligar "los métodos ortodoxos de investigación de campo con algún tipo de método experimental" para abordar el estudio de los sistemas políticos más allá de la teoría funcionalista imperante.

En efecto, en nuestra lectura, el aporte de Nadel correspondería a la dimensión propiamente teórica y metodológica, de modo de resolver respectivamente problemas relativos al estudio de la sociedad y, a través de ello, formalizar y validar el quehacer etnográfico. El aporte de Meyer, en cambio, pareciera tener-para la antropología contemporánea-un valor ilustrativo de un trasfondo no considerado en la formulación de la teoría propiamente tal, en el marco de la antropología clásica. Con el paso del tiempo se ha visto que, mientras la teoría para estudiar la sociedad pudo haber facilitado el desenvolvimiento del sistema colonial, el método comparativo ha permitido la liberación del quehacer antropológico de métodos estandarizados, tales como los propuestos por la teoría estructural-funcionalista que concebía a los sistemas políticos como preexistentes y reducidos a la función de reproducción de la sociedad. Por otra parte, la valoración de los contextos en los que emerge la teoría va teniendo en nuestros tiempos un valor cada vez más significativo, así como lo tiene también el focalizar la cuestión de la subjetividad y la reflexividad en el proceso de creación-de conocimiento (Haskell, Linds, e Ippólito, 2002). Al respecto los autores sostienen que "el conocer emerge no sólo desde o el interior de nuestras mentes, sino que emerge colectivamente a través del compromiso en una acción compartida.".

Nos interesa precisamente ilustrar este último proceso contextualizando de primera fuente una matriz operativa del quehacer antropológico que adquiere una connotación teórico-metodológica más que histórica, a pesar de situar los aportes en el tiempo. Esta matriz reconoce:

a) La existencia de presupuestos epistemológicos, teóricos, metodológicos y éticos que comprometen e involucran a los practicantes en el quehacer.

b) Las oportunidades disciplinarias y profesionales disponibles para aplicar este patrimonio en el contexto sociocultural del antropólogo.

C) Los desafíos derivados de la complejidad intrínseca del contexto en el cual el antropólogo se sumerge y es convocado a lograr un quehacer con sentido.

Esta matriz, por tanto, reconoce al sujeto con sus características personales, sus inquietudes y motivaciones, el papel de la socialización especializada y del contexto sociocultural y político en el que actúa, así como las exigencias de comunicación y acceso que este le plantea para su trabajo. Nuestro supuesto central es que sólo en los últimos cuarenta años ha sido posibie advertir la relación entre estos factores. Más aún, hoy sería posible situar al quehacer antropológico como objeto de análisis, lo que permitiría una mediatización o influencia reiativa de cada uno de ellos en la sociedad, de la cual el antropólogo forma parte. En otras palabras, sería posible sostener "cuán aplicado puede ser el conocimiento antropológico, como este debe ser, al referirse desde siempre al componente sociocultural de la vida humana."

En términos hipotéticos, proponemos que son las circunstancias o el contexto en el cual se construye el conocimiento, así como la reflexividad generada por el antropólogo y su comunidad de opinión, lo que incidiría de un modo significativo en el proceso de construcción de conocimiento antropológico. Al mismo tiempo, tal contexto constituirá la prueba posible de tal producto (dar vuelta)

Esta hipótesis debe reconocer la base epistemológica que la hace posible. Esta procede desde los enfoques interaccionistas y simbólicos (Goffman, Blumer, Mead, Geertz, 
entre otros) que asignan al contexto un rol fundamental en la construcción de conocimiento.

En la perspectiva histórica, la matriz anteriormente visualizada, permitiría sostener que el desarrollo de la disciplina ha sido, en general, estrictamente auto-referente y globalizante, pudiendo detectarse teorías y/o acercamientos mayormente provenientes del Primer Mundo. Este tipo de evolución de la disciplina se extiende hasta la actualidad, en que las discusiones filosóficas han marcado sus énfasis, particularmente en el campo de la historia de la ciencia y de la sociedad moderna, sea para refundar este estilo o para innovar desde él Es así como desde la segunda mitad del siglo pasado se han ido gestando perspectivas que, sin desdibujarse totalmente del modelo clásico, particularmente en el papel asignado a los referentes del corpus disciplinario, incursionen en replanteamientos teóricometodológicos en los que se asigna, como se afirmaba al inicio, importancia a la matriz operativa $y$, por ende, al contexto del desempeño. Reconocemos aquí, por ejemplo, a las antropologías de la diversidad en México, aunque no es esta la ocasión de abordar esta temática, de carácter general. Debemos concentrarnos en nuestro propio caso, en el que el contexto contestatario y al mismo tiempo consumista de la antropología nos ha llamado a una actitud de alerta. Esto quiere decir que el esfuerzo de esta oportunidad consiste en identificar un proceso local de construcción, el que con posterioridad podrá contrastarse con otros similares.

\section{Análisis y Discusión del quehacer antropológico en el contexto de la Antropología en la UCTemuco.}

En 1971 se crea el primer programa de Antropología Social y Cultural en el Sur de Chile, específicamente en la ciudad de Temuco. Esta institucionalidad emergente se sostiene en un corpus teórico - epistemológico que de un modo predominante direccionó el programa por los tres años en que se sostuvo el último gobiemo democrático pre - dictadura. Como ya se explicitara con anterioridad (Durán, 2002) el corpus teórico - epistemológico es caracterizable como postestructuralista y habría emergido en una sociedad del bloque socialista la cual, al igual que otras de gobierno dictatorial, generó una controversia al interior del país, particulamente, en el campo de las ciencias sociales, acerca del rol científico en relación a las políticas públicas. El tipo de coherencia del corpus teórico arrancó desde la redefinición del objeto estructural - funcionalista, hasta una particular vinculación con la institucionalidad universitaria, tras la búsqueda de espacios de autonomía en los procesos de transmisión y construcción de conocimientos. Así, mientras por un lado se problematizó la relación dialéctica entre el comportamiento individual y el societal, en el marco de condiciones socioculturales caracterizables, por otro, se inauguró un sistema de retroalimentación teórica y metodológica, tras la búsqueda de un marco reflexivo para acompañar el proceso socio institucional universitario. Con el paso del tiempo (tres décadas), hemos podido advertir que aunque este cambio de objeto representó un impacto destacable en la antropología chilena, el que no obstante no ha sido evaluado en profundidad, el cambio mismo se enmarcó en el modelo demostrado por Nadel.

En efecto, en los últimos años ha podido aclararse el contexto de emergencia de este acercamiento, aunque nos referiremos a él de un modo introductorio. En esta perspectiva, nos interesa mostrar, de un modo ilustrativo, cómo este acercamiento, conocido primero como teoría del reclutamiento (Stuchlik, 1976) y luego 
como teoría transaccionalista (Holy y Stuchlik, 1983), ha sido usado en nuestro contexto, por un lado, para construir conocimiento antropológico $y$, por otro, para analizar el proceso desde el enclave de su puesta en escena en el contexto local (Durán y Samaniego, 2003:59-77).

En efecto, a partir de la instalación de la teoría en el contexto nacional, se puede decir que ésta siguió dos principales caminos hacia la década de los '90: el de la academia -en la escuela de antropología de Temuco y en otra de Santiago-y en el mundo social-en el ámbito de las organizaciones solidarias en el contexto de la democracia re-naciente-. Dentro del campo universitario pueden apreciarse, a su vez, dos tendencias: una centrada en la práctica etnográfica vinculada a las teorías contemporáneas de la etnografía (Salinas, 2001) y otra que aborda el campo temático sociocultural del contexto multicultural y sociodiverso de La Araucanía (Durán y Berhó, 2003; Durán y Carrasco, 2004). A nivel general, mientras en las tres vertientes la etnografía interpretativa ha ocupado un papel central, en cada una de ellas se asoció a interpretaciones y a sustratos intelectuales y socio-éticos distintos. En el último caso (Dockendorff, 1993), la etnografía aparece como un medio para dilucidar qué de universal posee el concepto occidentai de "solidaridad" y qué manifestaciones particulares tiene en el contexto de las modernidades de las metrópolis. Esta etnografía se ve, por tanto, antecedida por una reflexión socio-ética y una finalidad sociopolítica y de participación ciudadana. En los otros dos casos, ya hemos planteado que la efnografía se constituye en el centro, pero en la academia se vive de distinto modo. En el caso de la antropología académica en Temuco, hoy día se advierte que las prácticas profesionales y disciplinarias y su respectiva distinción han estado teñidas no sólo por el contenido de la teoría y particularmente por el uso del método etnográfico contemporáneo, sino a su vez por la tendencia a convertir más y más las respectivas prácticas en materia de análisis del quehacer antropológico -como cualquier otro campo social-, aplicando la teoría de base.

\section{Prácticas y reflexividad en el quehacer antropológico en la UC Temuco.}

Continuando con el supuesto explicitado ariba, sostenemos la tesis de que es la reflexividad del antropólogo y, por tanto, de sus condiciones de posibilidad, la que se expresa en prácticas diferenciadas en tomo a un mismo tronco teórico $y$, por tanto, que es el foco de la reflexividad el que ilumina la búsqueda progresiva de entendimientos concernientes a la práctica profesional y antropológica, más allá del uso específico de la teoría. Esto quiere decir que, considerando el análisis de Comelles (1996), si bien en el contexto de Temuco se ha recurrido a la diferenciación entre profesión y disciplina y se ha logrado dimensionar el papel definitorio y al mismo tiempo limitado de la teoría, ha sido mayormente la academia la que se ha constituido en el espacio apto para ello. Este espacio es inherentemente social y por tanto está sometido a similares riesgos de forma que los ofrecidos por la sociedad y sus instituciones al practicante de la disciplina y de esta teoría. En otras palabras, desde el marco teórico se asume que la pervivencia en la academia es problemática y se requiere ganar nichos socioculturales en la medida en que las universidades se conciban como espacios de fuerzas sociales en contrastación.

En este sentido, y siguiendo a Comelles, no creemos que la profesionalización y s.u diferenciación con respecto al campo disciplinario constituyan en sí mismas el fin del quehacer, sino más bien pretendemos interpretarlas como espacios que exigen distintos desempeños en el marco de la teoría postestructurălista. En efecto, esta distinción carecería de una finalidad en la medida en que los profesionales de la antropología cultiven la disciplina sólo para ellos mismos 0 , en otras palabras, para la categoría de profesionales. Al revés, creemos que esta distinción permite establecer un vínculo reflexivo con la sociedad $y$, al mismo tiempo, con la disciplina, asumiéndola no como un "nido" de teorías sino 
más bien como espacios controversiales que condensan la multidimensionalidad de la realidad social como cualquier otro espacio social de esta naturaleza. Cuando en el campo profesional absorbemos las problemáticas sociales estamos sometiendo a la antropología a tener que dialogar con la sociedad, aceptando el modo institucionalizado de su logos, así como el abierto o creativo que descansa en una concepción de sociedad compuesta de seres con intencionalidades, estrategias y fines que otorgan sentido a su comportamiento. La teoría, en efecto, nos hace ver a la sociedad en sus diversos espacios institucionalizados en diversas formas y grados y nos plantea la posibilidad de optar para participar en ellos mediante estrategias sociales tan similares a las que puedan desarrollar otros actores sociales. Cuando nos situamos en el espacio de la disciplina, en tanto, estamos abstrayéndonos del pensamiento social para enfrentarnos al pensamiento intelectual $y / 0$ reflexivo con la posibilidad de vislumbrar finalidades y/o sustratos ético-sociales que la teoría por sí misma no incluye de un modo explícito. Lo anterior podría traducirse en el siguiente principio: Una teoría se hereda como patrimonio de interpretación de la disciplina, del mundo, de los hombres; la práctica reflexiva del antropólogo permite configurar distintos itinerarios reflexivos, no necesariamente contradictorios entre sí, que exigen establecer relaciones problemáticas con la sociedad y al mismo tiempo con la disciplina, en concordancia con las características del contexto local en que el quehacer se desarrolla.

A continuación presentaremos los avances que la teoría original del reclutamiento o transaccionalista ha fundado bajo la forma de su re-conceptualización y reinserción en el contexto local en el que se ha practicado, con posterioridad a su emergencia.

En una primera fase (1971 - 1992), las prácticas constituyeron eminentemente un esfuerzo por aplicar el paradigma teórico heredado. Los esfuerzos mayores se concentraron en intentar representar el paradigma, en cuya circunstancia, el problema social pasó a segundo plano. Eran prácticas orientadas a 'resolver' un problema más bien de conocimiento sugerido que demandado por el medio social. La teleología del conocimiento antropológico principal afirmaba que 'es posible el conocimiento antropológico por medio del acercamiento etnográfico', dado que esta metodología desvincula al sujeto del objeto de conocimiento a través de la teoría metodológica respectiva, pudiendo alcanzarse un tipo de cientificidad aceptable en la disciplina (Hammersley and Atkinson, 1994).

El impacto social de este tipo de quehacer ha estado limitado a los medios de divulgación y se ha manifestado por lo menos en dos niveles. Cuando se trataba de quehaceres demandados por instituciones, estas generalmente limitaban los resultados al ámbito de su competencia $y$, en general, los cuestionaban en la medida que la explicación proporcionada no suponía 'una solución' al problema, o sea, una respuesta comprensible para su manejo administrativo. Un segundo tipo de impacto emerge cuando la divulgación asume una forma corporativa y formal a través de seminarios y/o talleres. Estos se caracterizaron, en primer lugar, por reiterar el estilo 'explicativo' más que de orientación social de los estudios, y en segundo lugar, por la emergencia de la controversia acerca de la utilidad de la antropología y / 10 de la problemática social que ésta abordaba.

La segunda fase se fundó en la práctica cada vez más intensa del método de acceso etnográfico re-legitimado, que se funda en las corrientes que priorizan los acercamientos etnometodológicos y éticos, específicamente orientados a la temática del conocimiento tradicional (*Johnson, 1992). Aunque la etnografía contemporánea en uso se fundaba en gran medida en la construcción de representaciones, paulatinamente permitió la visualización de la presencia del "otro", sea en la forma del otro indígena o del otro social. El conocimiento antropológico en esta fase se torna más complejo al asumir de modo más pleno la diversidad sociocultural y las implicancias políticas, éticas e intelectuales de 
tal fenómeno. La lectura especializada de la época permite advertir que, mientras por un lado la teoría del transaccionalismo aprendida inicialmente era apta para llevar a cabo un análisis 'científico' (op.cit, 1976), por otro no permitía satisfacer las demandas del medio social sino sólo las del medio académico. El desempeño profesional, por un lado, debía emerger para responder a las demandas, y por otro, a las acusaciones o imágenes de la antropología asociadas a la inutilidad y a las actifudes colonialistas. Esta fase responde pues a esta demanda social explícita e implícita, incursionando en la teoría del diálogo de saberes (Ghiso 2000).

La tercera fase demuestra la fecundidad del acercamiento antropológico antes identificado, así como sus límites. Su relación con la teoría fundante, rica en matices analíticos e interpretativos, fortalece su argumentación tras cada práctica. Una demanda cada vez más compleja que representa la expresión de la estructura social en la región, otorga oportunidades emblemáticas para proponer una acercamiento teórico de alcance medio, la antropología interactiva, a través del cual se focaliza el compromiso teórico y social del antropólogo (Durán, 2002). Así, mientras los fundamentos teórico-metodológicos previnieron rigideces interpretativas y estimularon el sostenimiento de compromisos ético-sociales inherentes a las prácticas, promovieron nuevos desarrollos del paradigma culturalista. La formulación de un conocimiento 'intercultural' como un tipo de conocimiento propio de contextos multiétnicos y multiculturales, tipificable sólo mediante la identificación de factores concomitantes y no asociados a prácticas contingentes, constituyó un hallazgo teórico que ha continuado siendo problemáticamente fecundo (Durán et al, 2000). En el campo teórico, el acercamiento antropológico interactivo incorpora solidez al asociarse a los supuestos de los constructivistas radicales (Durán y Berhó, op. cit.), que niegan la certeza absoluta como en el neo-popperismo, pero garantizan la única veracidad posible a través de la construcción de "estructuras en tanto productos de nuestra propia y característica capacidad de representación" (Glasersfeld, 1998: 23). En otras palabras, se abre la posibilidad de un conocimiento en proceso: un conocimiento paradigmático en constante evolución.

La cuarta fase, que identificamos en la relación problemática entre una antropología periférica y una central y que presentáramos bajo la metáfora -"destejiendo telarañas"-(Durán 2005 AP 35-39), se hace presente en estado de crisis, es decir, en circunstancias en que debemos confrontarnos a las preguntas anteriormente formuladas que suponen, a su vez, evaluarnos respecto del legado originario, y en el marco de una dicotomía de poder. Para abordar esta fase recurriremos a la conceptualización que paraleliza las antropologías centrales respecto de las periféricas. Aceptamos esta nomenclatura reconociendo el supuesto de que la relación re-fundante entre antropología y sociedad, demostrable por los expertos del Primer Mundo, necesariamente posibilita un desempeño metodológico similar en una zona delimitable del Tercer Mundo: La Araucanía.

Nuestra reflexión concluye afirmando que el rasgo de perifericidad, como el término lo indica, es relativo $y$, lo que nos parece más importante, no es de carácter concluyente y por tanto no impide la vinculación significativa, por lo menos desde la periférica que nos representa. Esta propuesta es posible usando la ley de gravitación planteada por Cordero (1998) en el ámbito de la psiquiatría social, principio que en este caso se formularía como sigue: a cada sociedad le corresponde crear sus respectivos tipos de antropología y lo hará así como asuma los ámbitos intradiferenciados que la caracterizan. Se trata, por tanto, de una relatividad relativa (Gellner, 1993). Mediando el quehacer científico a la base, los niveles problemáticos analíticoexplicativos serán diferenciables en el contenido, pero no en la forma.

En la perspectiva de visualizar las 'contradicciones de nuestro tiempo', creemos un deber intentar referimos a "la manera en que deberíamos movernos" (ibid.: 105), lo que, en nuestro caso, concierne a clarificar los desempeños del rol del antropólogo. Al respecto sugerimos tomar en cuenta lo siguiente: 
1) la etnografía de la actuación del rol del antropólogo en la sociedad traza un tipo de antropología delimitada y/o delimitable por las condiciones sociales y políticas de ésta. En este contexto, el antropólogo se ve llevado a desempeñarse en una tensión entre actor social y especialista.

2) La experiencia y los logros de la disciplina muestran que esta tensión, si no se orienta, tiende a perjudicar al sujeto. Desde luego, no puede ser igual a un sentido común aprendido en tal estatus; el experto debe haber sido preparado para duplicar ese sentido común con otro (lbáñez, 1990).

3) El propio comportamiento social del antropólogo como persona y como profesional constituye la evidencia de que en la sociedad no puede darse un equilibrio perfecto entre conocimiento y acción, aun cuando la especialidad puede permitir visualizar una estrategia para el mejor aporte participativo.

4) En este juego de interacciones y planificaciones se advierte que la dimensión política propuesta por los expertos es parte constitutiva de la escena; se puede afirmar que esta dimensión es la que sitúa el estatus del antropólogo en la sociedad y, por tanto, su posibilidad de acción.

5) En el contexto anterior, la práctica y manejo de una ciencia "abierta y participativa" ha demostrado constituirse en una eficaz herramienta para contrarrestar y/o reforzar el estatus social con el de la especialidad.

6) En esta trama, los siguientes factores resultan relevantes:

a) El cultivo de la antropología debiera permitir el surgimiento de una personalidad analítica y amigable con la diversidad sociocultural, que lleve a la persona a aceptar voluntariamente el ejercicio de las dualidades y de sus respectivas articulaciones.

b) El ambiente de formación en antropología debiera favorecer el surgimiento de equipos de trabajo inter-generacionales, de modo de practicar la comunicación y particularmente la autocrítica y la crítica sana.

c) La formación antropológica debiera permitir el análisis permanente del desenvolvimiento sociopolítico y sociocultural de la sociedad local y global, con el objeto de advertir las tendencias predominantes y su impacto en las problemáticas que aborda, asi como la relación de tal dinámica con la evolución del pensamiento antropológico; $y$

d) Aunque el quehacer antropológico, sobre todo el periférico, constituye un foco de influencia en todo centro de enseñanza, los antropólogos deben estar conscientes de que su impacto estará circunscrito a las condiciones sociopolíticas imperantes en la sociedad $y$, en lo ético, a la calidad del acercamiento 'con la gente' que tal centro implemente y desarrolle y por el cual será evaluado en el largo plazo (Durán, 2005; Durán y Berhó, 2005).

\section{Conclusiones: aportes desde la teoría interactiva}

1. El modelo de evolución de la antropología del Primer Mundo ha sido monológico en la medida en que, sin estar ausente de reflexividad, ha sido producto de la propia academia o sector especializado que no ha dejado de observar a la sociedad en sus diversas manifestaciones a través del tiempo (Augé, 2004). Este tipo de producción ha sido eminentemente interpretativa de los movimientos y cambios socioculturales entre los diversos mundos que habitan la Tierra, así como de las problemáticas que subyacen o determinan la acción social. En este modelo no se advierte la influencia directa del contexto y sutilmente se deja ver la personalidad del antropólogo que opta por alguna teoría o enfoque. Este sería, pues, el modelo clásico transportado a la actualidad que incorpora temáticas contemporáneas y un posicionamiento develado del antropólogo en base mayormente a su productividad en el terreno intelectual. En zonas multiculturales e interétnicas como La Araucanía, en donde el 
quehacer antropológico ha ido formando parte de la escena y ha permitido la construcción de una imagen particular de la disciplina y de sus practicantes, el escenario es por tanto distinto y ofrece otras problemáticas por abordar. En efecto, aunque el antropólogo puede abstraerse de la dinámica social misma, es convocado por ella incluso por intereses pragmáticos $\mathrm{y} / \mathrm{o}$ de sobrevivencia, sentido a través del cual puede incluso instrumentalizar su aprendizaje. En el mejor de los casos puede optar por determinados escenarios socioculturales y moverse por orientaciones generales del conocimiento antropológico para abordar problemáticas de relaciones y/o de comunicaciones entre las distintas esferas sociales en las que se desenvuelve su quehacer. El antropólogo, en fin, puede desarrollar prácticas diversas, de corte metodológico o no, definidas por las distintas instituciones de la sociedad en su alcance y en su forma (Servicio PAIS, FOSIS, etc.). En este escenario, caracterizado por un tipo de relación cercana y lejana, se ha desarrollado un acercamiento interpretativo del quehacer antropológico que podría caracterizarse por su orientación identitaria y al mismo tiempo discontinuadora de la identidad global. Así, por ejemplo, se ha visto que la capacidad analítica del antropólogo puede percibir la complejidad que ofrece la sociedad contemporánea hoy día en América Latina, a partir de una constitución mono-cultural forzada incapaz de resolver las problemáticas sociales que ella misma genera, asumiendo que el propio quehacer antropológico es, del mismo modo, complejo y condicionado por tal trama social. En esta perspectiva, y para el caso ilustrado aquí, se revela que la discontinuidad con el modelo clásico no está en la renuncia al paradigma científico, sea este el hipotéticodeductivo o el naturalista, sino más bien en la posibilidad de asumir que estas herramientas heredadas se pueden relativizar a la hora de acentuar los vínculos identitarios con la disciplina y con la sociedad. En efecto, la academia o el pensamiento intelectual occidental ha elaborado en el tiempo un arsenal de dispositivos teóricos de distinto orden -filosóficos, psicológicos, políticos, etc.-, pero este arsenal no cobra sentido en el quehacer antropológico hasta que las prácticas asi lo exijan. Lo mismo ocurre con respecto a los fines, es decir, respecto del para qué de la antropología, a partir de un dónde. Se ha ilustrado aquí que la teoría de la antropología interactiva es doblemente dialógica: así, está re-encontrándose con la base ontológica y ética de que la comunicación humana necesariamente es dialógica $y$, al mismo tiempo, está descubriendo que su finalidad, en superación de un cómo, debe concordar con las finalidades que la sociedad y la academia también han definido, pero que no necesariamente explicitan. En este sentido, la teoría interactiva debe dialogar con la productividad intelectual y la social sin abandonar el uso reflexivo de sus prácticas. Antes bien, debe reformular un quehacer antropológico distinto que podríamos denominar como periférico y que actualmente se presenta en oposición valórica respecto de la hegemonía social del modelo academicista y o científicoclásico.

2. En concordancia con lo anterior, la incursión en el campo de la marginalidad social extrema permite demostrar la importancia de la perspectiva antropológica en el entendimiento de sus bases socioculturales (Berho, 2004), detectándose a su vez la carencia de enfoques que faciliten la comprensión de otras dimensiones asociadas al mismo campo. Al respecto, sostenemos que la antropología debe buscar aquellas explicaciones que ayuden a situar de mejor forma las prácticas contextuales, visualizando en este caso, por una parte, el papel de la economía y la estructura del trabajo $y$, por otro, el de los distintos modelos culturales y políticos que la sociedad ofrece para interpretar y tratar el problema social. Esto no significa perder de vista que estos referentes otorgarán mayor coherencia al quehacer explicativo del antropólogo, pero no necesariamente al piano interactivo, que responde a la dinámica de la organización social. En este caso, se tiende, por un lado, a consumar la profecía sociologizante en el sentido de que los sujetos se demuestran determinados por el modo en que las estructuras se hacen presentes en su cotidianeidad, pero por otro lado-conscientemente o no- "luchan" por 
liberarse de tales profecías al movilizar modelos de diverso origen -religiosos, éticos, políticos y, en menor medida, científicos- para enfrentar el problema social. De aquí la necesidad de adquirir un posicionamiento ético-social respecto de los problemas sociales vividos por la gente y asumidos desde el quehacer profesional y disciplinario. Nuestra asunción de fondo es que un antropólogo actúa mejor en los contextos latinoamericanos si es capaz de sortear las demandas de la interacción cara a cara, haciendo que su quehacer sea demandado o que ayude a visibilizar las problemáticas sociales y su forma de abordaje y que, al mismo tiempo, dialogue con la base ético-filosófica de la teoría en tanto componente del constructo mayor de la ciencia respecto de referentes ético-sociales.

La antropología de la alimentación emergente en Temuco durante el último año, ha permitido a su vez demostrar que el modelo interactivo funde la voluntad de participar en los procesos de transformación social -propia de la antropología del desarrollo- con enfoques deconstruccionistas que indagan en las tramas de conocimiento y poder involucradas en tales procesos. El desplazamiento de los paradigmas analíticos, desde las estructuras estáticas hacia los procesos e interacciones de naturaleza cultural, sitúa a la antropología interactiva en un punto de encuentro entre perspectivas explicativas y empáticas con la comunicación, los significados y los símbolos. Nuestra hipótesis teórica a este respecto es que el fenómeno de la alimentación humana es dependiente tanto de estructuras sociales como de procesos culturales de asignación de significados, y tanto la 'buena' como la 'mala' alimentación dependerán de la combinación de estos dos factores. Thomas ha dicho que los actuales enfoques antropológicos "hacen más justicia que hace treinta años" tanto con los determinantes de la vida social, como con las capacidades de los individuos para moldear sus propias circunstancias de vida (Thomas, 1997: 14). Reconoce en esta característica cierto riesgo a la exageración de lo particular de cada contexto, en oposición a los excesos universalistas de las generaciones anteriores. En el ámbito del desarrollo y los problemas alimentarios, desde la perspectiva antropológica interactiva estamos abordando la posibilidad de construir una alternativa a dicha polaridad. La antropología interactiva y su principio de 'vínculo consciente' con sus contextos de trabajo, permite reformular la pregunta etnográfica clásica al verse enfrentada al nuevo desafío de describir y presentar lo encubierto y lo implícito en los procesos de intervención alimentaria a través de un lenguaje analítico explícito. Ya no pregunta a la gente ¿̇qué?, ¿̇cómo? y àpor qué?, sino żcómo entiende lo que come? Sitúa a la gente en un nuevo rol, el de co-intérprete de su propia realidad. El aporte de esta antropología interactiva de la alimentación consiste, entonces, en descubrir el trasfondo de los problemas alimentarios e identificar los diferentes puntos de vista que están participando de su dinámica, tanto en el plano social como en el plano analítico (Carrasco, 2005).

En el campo de las relaciones interétnicas, y de un modo similar a los anteriores en la forma, pero diferente en el fondo, el desafío de las prácticas antropológicas es intentar dialogar o reconocer sus dificultades en contextos culturalmente diversos e inconmensurables. El análisis de las prácticas ha indicado la necesidad de reconocer que la interactividad no puede seguir la misma forma que en el ámbito de la marginalidad, ya que en este caso debe duplicar el contenido cultural de las estrategias de un modo similar al propuesto en el campo de la alimentación. En el plano social, la antropología se ve obligada profesional y disciplinariamente hablando a crear espacios de intermediación (Durán y Berho, 2004; Catriquir y Durán, 2004) que posibiliten la diferenciación y la búsqueda de acuerdos organizacionales finitos sin pretender necesariamente alcanzar el ideal hermenéutico de la fusión de horizontes (Gadamer, 1992). En efecto, los avances progresivos que se han hecho en torno a la teoría de la diversidad desde la perspectiva de la antropología interactiva revelan que la teoría por sí misma no resuelve el sentimiento que genera la vivencia traumática de la diversidad cultural. En este campo, el antropólogo tiene que disponer de un dispositivo filosófico que 
otorgue sentido a su quehacer, del mismo modo que plantee y promueva propuestas que, siendo derivadas de la interacción profunda con los actores sociales, permita un horizonte de cambio sociocultural en el tiempo. Cuando la teoría ha llamado la atención respecto de la construcción y reconstrucción de procesos vulnerados y en riesgo de alteración profunda, la antropología interactiva debe abordar la compleja tarea de participar en las políticas sociales responsables de dicho deterioro y al mismo tiempo intentar asumirlo y buscar formas, en conjunto con los actores directamente involucrados, para proyectar el cambio cultural, sea visibilizando la problemática o sumándose creativamente a las propuestas en marcha.

\section{Bibliografía}

AUGÉ, M., 2004. ¿2Por qué vivimos? Por una antropología de los fines. Gedisa, Barcelona.

BERHÓ, M., 2004. Antropología de la marginalidad extrema. Una propuesta local. En Antropología y sociedad, ANTHROPOS № 207. Barcelona (en preparación).

CATRIQUIR, D.YT.DURÁN, 2004. Günezuamgerpun mew epu xokiñ rakizuam. Abriendo cauces de con-versación entre pensamientos. En Antropología y sociedad, ANTHROPOS $N^{\circ} 207$. Barcelona (en preparación).

COMELLES, 1., 1996, Las profesiones y el estado. En Ensayos de antropología cultural, J. Prats y A. Martínez (Eds.), pp. 173-183. Ariel S.A., Barcelona.

CORDERO, M., 1998. Salud mental y psiquiatría: el modelo gravitacional. Universidad de Santiago de Chile, LOM, Santiago.

DOCKENDORFF, C., 1993. Solidaridad: La construcción social de un anhelo. Soles, Santiago.

DURÁN, T., 2002. Antropología interactiva: un estilo de antropología aplicada en la IX Región de La Araucanía, Chile. CUHSO, Volumen VI, Nº 1:23-57.
DURÁN, T. Y M. BERHO, 2003. Antropología interactiva: consciencia y práctica dual del rol del antropólogo en una sociedad multiétnica y multicultural. CUHSO, Volumen VII, $N^{\circ} 1: 34-48$.

DURÁN, T. Y M. BERHO, 2004. Modelos y prácticas socioculturales en torno a la interculturalidad. Bases fundantes. En Rostros y fronteras de la identidad, M. Samaniego y C. G. Garbarini (compiladores), pp. 119-149. Pehuén, Santiago.

DURÁN, T. Y D. CATRIQUIR, 2004. Complejidades en los estudios avanzados para abordar la diversidad cultural: una introducción a la metodología transcultural. Presentado en el VI Congreso Latinoamericano de Educación Intercultural Bilingüe, Temuco.

DURÁN, T. Y N. CARRASCO, 2004. Critical review of the ecosystem approach to human health: the chilean - mapuche interethnic context. Review Ecohealt, Springer - Verlag, New York. (Comité Editorial)

DURÁN, 2005. Duplicando la antropología en la araucanía de Chile. Revista Atrophos $N^{\circ} 207$, Barcelona-España.

JOHNSON, M. 1992 Capturing traditional environen talknowledgl. Editorial LORE. JDRC Canadá.

GADAMER, H. G., 1992. Verdad y método. Sígueme, Salamanca.

GELLNER, E., 1993. Encuentros con el nacionalismo. Alianza, Madrid.

GLASERSFELD, E., 1998. Despedida de la objetividad. En El ojo del observador. Contribuciones al constructivismo, P. Watzlawick y P. Krieg (Comps.), Gedisa, Barcelona.

HAMMERSLEY, M. YP.ATKINSON, 1994. Etnografía. Paidós, Barcelona. 
HOLY, L. Y M. STUCHLIK, 1983. Actions, norms and representations. Foundations of anthropological inquiry. Cambridge University Press, Great Britain.

IBÁÑEZ, J., 1990. El regreso del sujeto. La investigación social de segundo grado. Amerinda, Santiago de Chile / Siglo XXI, Madrid.

NADEL, S., 1966. Teoría de la estructura social. Guadarrama, Madrid.

SALINAS, F., 2001. El bario Yungay de Santiago. Apuntes etnográficos. Universidad Bolivariana, Santiago.

STUCHLIK, M., 1976. Life on a half share. Mechanisms of social recruitment among the mapuche of southern Chile. C. Hurst \& Company, London.

THOMAS, N., 1997. Epistemologías de la antropología. Revista Internacional de Ciencias Sociales $N^{\circ}$ 153. UNESCO. Disponible en http://www.unesco.org/issj/rics 153/thomaspa. html. 